\title{
Scaling of submonolayer island sizes in surfactant-mediated epitaxy of semiconductors
}

\author{
Vasily Cherepanov, Sergey Filimonov, Josef Mysliveček, and Bert Voigtländer* \\ Institut für Schichten und Grenzflächen (ISG 3) and cni-Center of Nanoelectronic Systems for Information Technology, \\ Forschungszentrum Jülich, 52425 Jülich, Germany
}

(Received 16 March 2004; published 5 August 2004)

\begin{abstract}
We study submonolayer island size distributions in the epitaxy of $\mathrm{Si}$ and $\mathrm{Ge}$ on the $\mathrm{Si}(111)$ surface with $\mathrm{Bi}$ as a surfactant. We show that sizes of Si islands at different growth temperatures scale to a standard scaling function that peaks at the mean island size. Size distribution of Ge islands demonstrates qualitatively different behavior: With the decreasing temperature the most probable size in the population of Ge islands shifts towards small island sizes so that the peaked scaling function degenerates to a decreasing one. The observed scaling phenomena are found to be inherent to the mechanism of growth which involves exchange and de-exchange processes of deposited atoms with the surfactant and the strong passivation of step edges in the presence of the surfactant.
\end{abstract}

DOI: 10.1103/PhysRevB.70.085401

PACS number(s): 68.55.Ac, 68.47.Fg, 81.15.Hi, 81.15.Aa

\section{INTRODUCTION}

Experimental investigations of density and size distribution of submonolayer island populations yield qualitative and in certain cases even quantitative information regarding microscopic mechanisms that determine the growth of the system under scrutiny. One particular type of information is whether the studied growth system clasifies within a standard growth model or not. In the standard growth model, ${ }^{1}$ atoms arrive at a substrate with a flux $F$ and diffuse on the substrate with a temperature dependent diffusion rate $D$. No desorption of atoms from the surface is allowed. When two or more diffusing atoms meet a two-dimensional (2D), one monolayer (ML) high island nucleates and grows further by the diffusion limited capture of adatoms. In this way a population of 2D islands covering a fraction $\theta$ of the surface develops. In the precoalescence regime of growth the island population is characterized by the island size distribution $N_{s}$. For various amounts of deposited material expressed in terms of the surface coverage $\theta, N_{s}$ scales onto a single function $f(x)$ :

$$
N_{s}=\theta /\langle s\rangle^{2} f(s /\langle s\rangle),
$$

where $\langle s\rangle$ denotes the mean island size. ${ }^{2}$ In the standard growth model $f(x)$ is a peak function with a peak at $x=1 .^{2-5}$ In addition, the total island density $N$ is a power law of $F$ and $D:^{1}$

$$
N \propto(F / D)^{\chi}, \quad 1 / 3 \leqslant \chi<1 .
$$

When the above conditions are met, predictions of the standard growth model can be used to measure $D$ and other model parameters. ${ }^{6,7}$

On the other hand, the experimentally observed deviations from the behavior predicted by Eqs. (1) and (2) can be used to identify important growth mechanisms beyond the standard model. Examples include observations of growth with $\chi=0$ in systems with post-deposition ${ }^{8}$ or displacive ${ }^{9,10}$ nucleation, occurrence of a decreasing scaling function $f(x)$ caused by these mechanisms ${ }^{8-10}$ or by a strong anisotropy of the surface structure, ${ }^{11}$ growth with $\chi>1$ in systems with hindered incorporation of atoms into the islands, ${ }^{12,13}$ and the observation of a multipeak island size distribution when stable islands of "magic" sizes exist. ${ }^{14}$ Additionally, transition from a peaked scaling function to a decreasing one with the increasing growth temperature was predicted theoretically for systems with prominent desorption of deposited particles. ${ }^{15,16}$

In surfactant mediated epitaxy (SME) ${ }^{17-21}$ particles of the deposited material arrive at a surface, where a monolayer of another species, so called surfactant, is adsorbed. This modifies the growth scenario compared to the growth without surfactant, allowing, e.g., layer-by-layer growth of relaxed layers in highly strained Ge/Si heteroepitaxy. ${ }^{18,19}$ Therefore SME systems are good candidates to have a behavior different from that predicted by the standard growth model. Indeed, $\chi>1$ was measured in submonolayer growth of Ge on the $\mathrm{Pb}$-covered $\mathrm{Si}(111)$ surface. ${ }^{22}$ Meanwhile, no measurements of the island size distributions in SME have been reported so far.

In this work, we present the first experimental study of submonolayer island size distributions in SME. We use a scanning tunneling microscope (STM) to observe the morphology of $\mathrm{Si}$ and Ge layers grown on $\mathrm{Si}(111)$ by $\mathrm{SME}$ with $\mathrm{Bi}$ as a surfactant (Bi-SME). We find scaling of $N_{s}$ according to Eq. (1) for both $\mathrm{Si}$ and $\mathrm{Ge}$. For Si, a standard scaling function is observed. For $\mathrm{Ge}$, a scaling function with a peak strongly shifted towards small island sizes is found. This nonstandard scaling is temperature dependent: for higher temperatures, the scaling function approaches the standard one. We demonstrate, that the observed scaling phenomena can be explained considering exchange and deexchange processes of deposited atoms with surfactant and the passivation of step edges in the presence of surfactant. ${ }^{23}$ For this purpose, we perform kinetic Monte Carlo (KMC) simulations of a generalized diffusion-de-exchange-passivation (DDP) model of surfactant mediated epitaxy. ${ }^{24}$ In terms of the DDP model, stronger passivation of the step edges for Ge atoms than for $\mathrm{Si}$ atoms is required to obtain the experimentally observed difference of the submonolayer scaling in Ge and $\mathrm{Si} \mathrm{Bi}-\mathrm{MBE}$ on the $\mathrm{Si}(111)$ surface. 


\section{EXPERIMENT}

Experiments were performed in an ultrahigh vacuum chamber with a base pressure $<5 \times 10^{-11}$ Torr. $\mathrm{Si}(111)$ substrates doped to $1 \times 10^{19} \mathrm{~cm}^{-3} \mathrm{Sb}$ were resistively heated by passing dc current. Standard flashing procedure yielded clean $\operatorname{Si}(111) 7 \times 7$ surface. This surface was kept at $500{ }^{\circ} \mathrm{C}$ and terminated by $\mathrm{Bi}$ evaporated at a rate $1 \mathrm{BL} / \mathrm{min}(1 \mathrm{BL}$ $=1.56 \times 10^{15}$ atoms $/ \mathrm{cm}^{2}$ ) from a Knudsen cell. After Bi termination, substrate temperature was set to a desired level and $\mathrm{Ge}$ or $\mathrm{Si}$ was deposited from a graphite or tantalum crucible, respectively, both heated by electron bombardment. Bi was codeposited at a rate $1 \mathrm{BL} / \mathrm{min}$ and the characteristic $\mathrm{Bi}$ induced $\sqrt{3} \times \sqrt{3}$ surface structure was maintained during growth.

Scaling of $N_{s}$ was studied for layers grown at $F=0.2 \mathrm{BL} / \mathrm{min}$ and $\theta$ from $0.06 \mathrm{BL}$ to $0.2 \mathrm{BL}$. Substrate temperatures were set to $440{ }^{\circ} \mathrm{C}$ for Ge layers and $480{ }^{\circ} \mathrm{C}$ for $\mathrm{Si}$ layers to obtain a comparable island density in both systems. Typical surface morphology of a $\mathrm{Ge}$ and a Si layer is shown in Figs. 1(a) and 1(b). Surface morphology of the Ge layer distinctly differs from that of the Si layer: islands of different sizes, among them many small islands are observed in the Ge layer, whereas in the Si layer the island sizes are more uniform.

This observation is further supported by a comparison of the island size distributions $N_{s}$ for Ge and $\mathrm{Si}$ that are shown in Figs. 1(c) and 1(d), respectively. ${ }^{25}$ In both cases, we observe a fit of the rescaled $N_{s}$ for different $\theta$ onto a single scaling function. The scaling function peaks at $s=\langle s\rangle$ for $\mathrm{Si}$, however, the peak is found at $s<0.2\langle s\rangle$ for Ge. Thus, Ge SME on Bi-terminated $\mathrm{Si}(111)$ is not described by the standard nucleation model. For both $\mathrm{Ge}$ and $\mathrm{Si}$, additional measurements were performed at complementary temperatures $480{ }^{\circ} \mathrm{C}$ for $\mathrm{Ge}$ and $440{ }^{\circ} \mathrm{C}$ for $\mathrm{Si}$. These reveal, that $N_{s}$ for $\mathrm{Ge}$ approaches a normal one with increasing temperature [Fig. 1(c)]. $N_{s}$ for Si does not change [Fig. 1(d)].

It is well established that the island size distribution is rather sensitive to the spatial correlations of the island locations. ${ }^{26}$ A measure of the spatial arrangement of islands in the experiment is the nearest-neighbor separation distribution $N(r) .{ }^{27}$ This quantity represents the density of islands having its nearest neighbor at a distance $r$. It scales as $N(r)$ $=N g(r /\langle r\rangle)$ where $\langle r\rangle$ denotes a mean distance to a nearest neighbor. Rescaled $N(r)$ for Ge and $\mathrm{Si}$ are shown in Figs. 1(e) and 1(f). In contrast to the Si case the rescaled $N(r)$ is asymmetric for Ge with the peak shifted toward smaller island sizes. This is an indication of a certain loss of spatial correlation $^{27}$ between $\mathrm{Ge}$ islands compared to $\mathrm{Si}$ islands. The islands can nucleate at smaller relative separations in the $\mathrm{Ge}$ layer than in the Si layer. A similar effect can be observed at preexisting step edges on the $\operatorname{Si}(111)$ substrate, as seen in Fig. 2. Islands can nucleate very close to the step edge in the Ge layer [Fig. 2(a)]. On the contrary, in the Si layer a pronounced denuded zone ${ }^{28}$ free of islands appears [Fig. 2(b)].

To complete the experimental picture of $\mathrm{Ge}$ and $\mathrm{Si} \mathrm{Bi}$ SME on the $\operatorname{Si}(111)$ surface, we measured the exponent $\chi$ of Eq. (2). The value of $\chi$ for Ge and Si was determined from values of $N$ obtained at five different settings of $F$. $F$ was
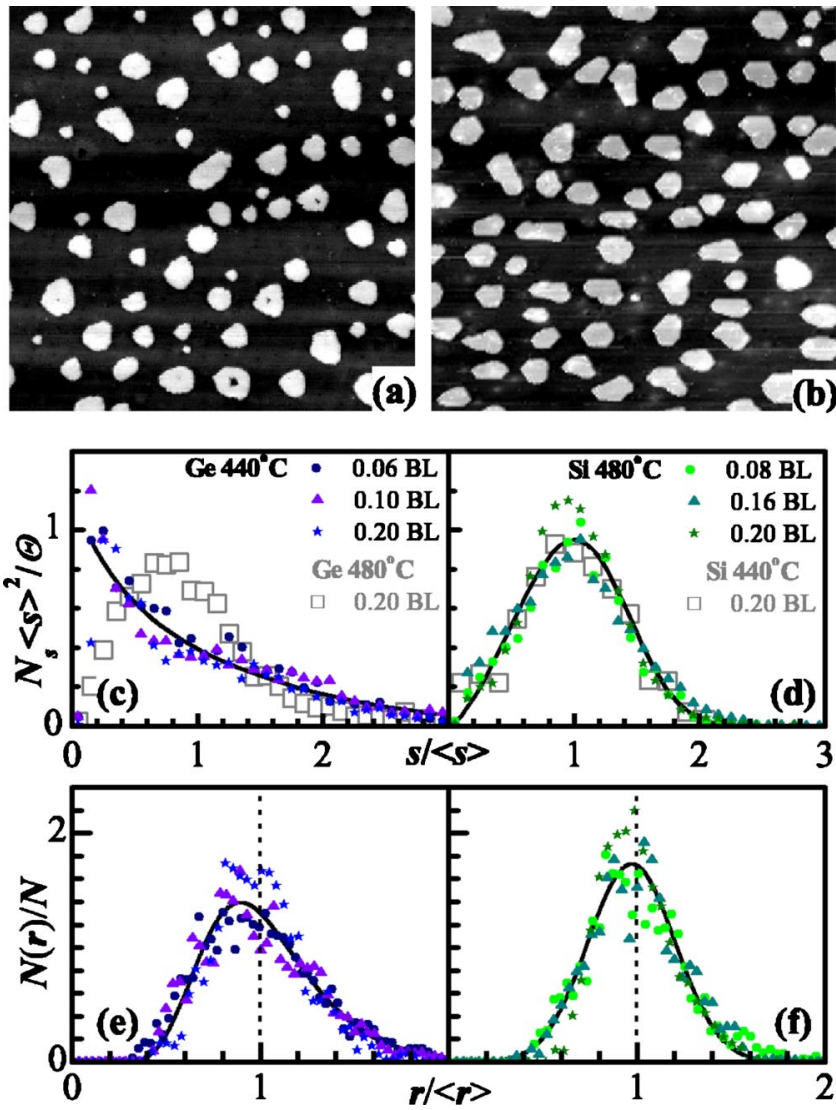

FIG. 1. (Color online) Morphology of (a) Ge layer, (b) Si layer grown by $\mathrm{Bi}-\mathrm{SME}$ on the $\mathrm{Si}(111)$ surface. $\theta=0.2 \mathrm{BL}, \quad F$ $=0.2 \mathrm{BL} / \mathrm{min} . T=440{ }^{\circ} \mathrm{C}$ for $\mathrm{Ge}, T=480{ }^{\circ} \mathrm{C}$ for Si. Image width is $210 \mathrm{~nm}$ in (a), $140 \mathrm{~nm}$ in (b). (c), (d) Corresponding plots of the rescaled $N_{s}$ for various $\theta$ (closed symbols), measurement of $N_{s}$ for complementary temperatures (open symbols). (e), (f) Corresponding rescaled nearest neighbor separation distribution $N(r)$ of islands in Ge and Si layer. Lines represent guides to the eye (Ref. 42).

varied between $0.08 \mathrm{BL} / \mathrm{min}$ and $6.3 \mathrm{BL} / \mathrm{min}, \theta$ was set to $0.2 \mathrm{BL}$ and $T$ to $440^{\circ} \mathrm{C}$ for both $\mathrm{Ge}$ and Si. We obtain similar values $\chi_{\mathrm{Ge}}^{440}{ }^{\circ} \mathrm{C}=0.37 \pm 0.02$ for $\mathrm{Ge}$ and $\chi_{\mathrm{Si}}^{440}{ }^{\circ} \mathrm{C}$ $=0.36 \pm 0.02$ for $\mathrm{Si}$, within standard limits for $\chi$.

The observed nonstandard scaling in Bi-SME of Ge on $\mathrm{Si}(111)$ differs from all observations of nonstandard sub-
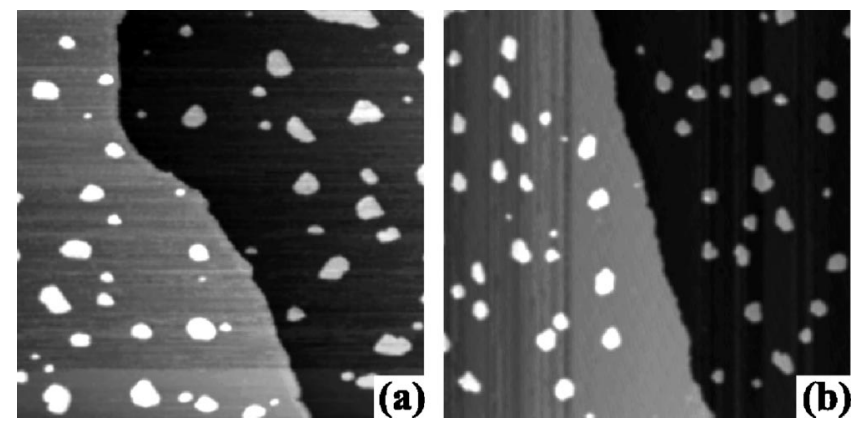

FIG. 2. Island population near a step edge in (a) Ge layer, (b) $\mathrm{Si}$ layer grown by $\mathrm{Bi}-\mathrm{SME}$ on the $\mathrm{Si}(111)$ surface. $\theta=0.1 \mathrm{BL}, F$ $=0.2 \mathrm{BL} / \mathrm{min}, T=440{ }^{\circ} \mathrm{C}$ for Ge, $\theta=0.08 \mathrm{BL}, F=0.2 \mathrm{BL} / \mathrm{min}, T$ $=480{ }^{\circ} \mathrm{C}$ for Si. Image width is $230 \mathrm{~nm}$ in (a), $130 \mathrm{~nm}$ in (b). 
monolayer scaling mentioned above. The observed scaling function for $N_{s}$ is not multipeak. ${ }^{14}$ Rather, it can be approximated by a decreasing function [Fig. 1(c)]. Such a behavior cannot be related to post-deposition ${ }^{8}$ or displacive ${ }^{9,10}$ nucleation, because a nonzero exponent $\chi_{\mathrm{Ge}}^{440}{ }^{\circ} \mathrm{C}$ is observed. Arguments on pronounced anisotropy ${ }^{11}$ do not apply to the $\sqrt{3}$ $\times \sqrt{3}$ structure of the Bi-terminated $\mathrm{Si}(111)$ surface. A transition between the peaked and decaying scaling functions due to desorption ${ }^{15,16}$ can be excluded as the expected temperature dependence for this desorption-induced phenomenon is opposite to that observed in our study.

We will not consider eventual strain contributions to the scaling phenomena observed in our experiments. The strain contributions to submonolayer island scaling have so far been detected only in semiconductor heteroepitaxy on surfaces with pronounced anisotropy, in particular, by evaluating the distribution of projections of island sizes onto two nonequivalent directions..$^{29,30}$ In addition, the observed difference between the rescaled $N_{s}$ of a strained system [Ge on Bi-terminated $\mathrm{Si}(111)]$ and an unstrained system [ $\mathrm{Si}$ on $\mathrm{Bi}$ terminated $\mathrm{Si}(111)]$ is much larger than the effects of strain on the scaling of $N_{s}$ predicted for isotropic systems. ${ }^{31,32}$

\section{DIFFUSION-DE-EXCHANGE-PASSIVATION MODEL OF SME}

The deviation of the scaling observed in Bi-mediated growth of $\mathrm{Ge}$ on $\mathrm{Si}(111)$ from the predictions of the standard growth model justifies a search for additional atomic-scale processes which could contribute to the observed scaling phenomena. In the following, we will study the scaling of $N_{s}$ in the diffusion-de-exchange-passivation model of SME proposed in Refs. 23 and 24.

The DDP model assumes three basic processes that happen during the SME growth (Fig. 3): diffusion of deposited atoms on top of the surfactant, exchange of material atoms with surfactant to incorporate below the surfactant layer, and de-exchange of material atoms with surfactant atoms to get back on top of the surfactant. Processes are considered to be thermally activated with rates $\nu_{i}$ having an Arrhenius form $\nu_{i}=\nu_{0} \exp \left(-E_{i} / k_{B} T\right)$, where $\nu_{0}$ is the common prefactor of the order $10^{13} \mathrm{~s}^{-1}, k_{B}$ is the Boltzmann's constant, $T$ temperature, and $E_{i}$ the activation energy of the $i$ th process with $E_{D}$, $E_{\mathrm{ex}}, E_{\mathrm{dex}}$ standing for diffusion, exchange and de-exchange processes, respectively. Generally, $E_{\mathrm{dex}}>E_{\mathrm{ex}}$, to account for the increase of the binding energy of single material atoms upon incorporation. Important is a definition of the behavior of material atoms at step edges. In the DDP model, not only terraces but also step edges are passivated, i.e., incorporation of atoms into step edges does not happen automatically. Upon incorporation of an atom at the step edge, its binding energy increases more than that of an atom on the terrace. Accordingly, $E_{\mathrm{dex}}$ at step edges is larger than $E_{\mathrm{dex}}$ for staying alone atoms.

The above described DDP model was used to show, that the island density $N$ in SME depends on a combination of $E_{D}, E_{\mathrm{ex}}, E_{\mathrm{dex}},{ }^{24}$ rather than on $E_{D}$ only as is in the standard model. ${ }^{1}$ Thus, observations of $N$ in SME are not directly related do the $E_{D}$ as was expected in earlier works. ${ }^{20,21}$ It has

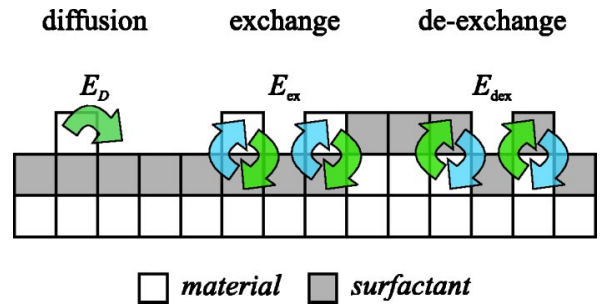

FIG. 3. (Color online) Growth processes considered in the DDP model of SME (Refs. 23 and 24). Related activation energies of these processes $E_{D}, E_{\mathrm{ex}}, E_{\mathrm{dex}}$ generally differ for material atoms in plane and material atoms at step edges.

also been demonstrated, ${ }^{23}$ that assuming a proper strain dependence of $E_{\mathrm{dex}}$ at step edges, the experimentally observed transition from 2D growth at low temperatures to 3D growth at high temperature ${ }^{20}$ can be explained.

To study scaling of submonolayer island size distributions we generalized the DDP model in terms of the well established bond-counting KMC scheme of epitaxial growth, ${ }^{3,4}$ which is well suited for such purposes. In this scheme, the activation energy of a growth process for a particular atom depends on a number $n$ of its nearest lateral neighbors. Thus, in the DDP model, the activation energy for diffusion of atoms on top of the surfactant becomes $E_{D}=E_{s}+n_{\text {top }} E_{n}^{\text {top }}$, where $E_{s}$ is the activation energy for hopping of a single atom on top of the surfactant, and $n_{\text {top }} E_{n}^{\text {top }}$ is the increase of the binding energy of an atom before a hop due to $n_{\text {top }}$ nearest lateral neighbors. Additional energy barriers to hopping of atoms across the step edges ${ }^{33}$ are not considered. For simplicity, no difference between surfactant and material neighbors on top of the surfactant is made. The activation energy for exchange $E_{\text {ex }}$ is kept constant independent of $n_{\text {top }}$, which ensures the passivation of the step edges in the DDP model. $^{23,24}$

Due to incorporation below surfactant, atoms increase their binding energy by $E_{\mathrm{inc}}+n_{\mathrm{inc}} E_{n}^{\mathrm{inc}}$ with respect to single atoms on top of the surfactant. The linear term $n_{\text {inc }} E_{n}^{\text {inc }}$ represents the step edge contribution to the binding energy, $n_{\text {inc }}$ is the number of nearest neighbors of an atom that are incorporated below surfactant. The de-exchange happens with an activation energy $E_{\mathrm{dex}}=E_{\mathrm{ex}}+E_{\text {inc }}+n_{\text {inc }} E_{n}^{\text {inc }}-n_{\text {top }} E_{n}^{\text {top }}$ which accounts properly for the difference of binding energies of an atom that is determined by $n_{\text {inc }}$ before and $n_{\text {top }}$ after deexchange.

We employed an unrestricted solid-on-solid simulation scheme on a square lattice with a periodic boundary condition. ${ }^{3,4}$ Simulation events have been selected with a standard algorithm. ${ }^{34}$ To save the computation time we set $F$ and $T$ to values that yield island sizes approximately 20 times smaller compared to those in the experiment. The parameters $E_{s}, E_{\mathrm{ex}}$, and $E_{\text {inc }}$ determining the behavior of single atoms in the DDP model have been selected in accord with $a b$ initio calculations of the activation energies for hopping, exchange, and de-exchange of single $\mathrm{Si}$ and $\mathrm{Ge}$ atoms on the As-terminated $\mathrm{Si}(111)$ surface. $^{35}$

The major qualitative predictions of Ref. 35 can be summarized as follows. First, the activation energies $E_{s}$ for hopping are equal in both systems $(0.25 \mathrm{eV})$. Second, the acti- 
vation energy $E_{\mathrm{ex}}$ for exchange of a single $\mathrm{Si}$ atom $(0.27 \mathrm{eV})$ is close to the hopping barrier $E_{s}$, i.e., $\mathrm{Si}$ atoms easily incorporate under the surfactant layer. In contrast, Ge atoms have to overcome a significantly higher exchange barrier $(0.71 \mathrm{eV})$ and therefore they can stay longer on top of the surfactant. Third, the activation energies for de-exchange $E_{\mathrm{dex}}=E_{\mathrm{ex}}+E_{\mathrm{inc}}$ are, in fact, comparable for both materials ( $1.07 \mathrm{eV}$ for $\mathrm{Si}$ and $0.9 \mathrm{eV}$ for $\mathrm{Ge}$ ). Assuming a similar qualitative behavior on the Bi-terminated $\mathrm{Si}(111)$ surface $^{36}$ we used two different sets of parameters in our KMC simulations of Si and Ge deposition: $E_{s}=0.3 \mathrm{eV}, E_{\mathrm{ex}}=0.3 \mathrm{eV}, E_{\mathrm{inc}}$ $=0.3 \mathrm{eV}$ for $\mathrm{Si}$, and $E_{s}=0.3 \mathrm{eV}, E_{\mathrm{ex}}=0.5 \mathrm{eV}, E_{\mathrm{inc}}=0.1 \mathrm{eV}$ for Ge. The binding energies $E_{n}^{\text {top }}$ and $E_{n}^{\text {inc }}$, as well as the attempt frequency $\nu_{0}$ were set to be equal for both materials with $E_{n}^{\text {inc }}=0.25 \mathrm{eV}, E_{n}^{\text {top }}=0.05 \mathrm{eV},{ }^{37}$ and $\nu_{0}=2 \times 10^{12} \mathrm{~s}^{-1}$. To clearly identify one of the possible reasons for the experimentally observed difference in scaling of $N_{s}$ in $\mathrm{Ge}$ and $\mathrm{Si}$ Bi-SME on $\mathrm{Si}(111)$, the activation energies in our KMC simulation differ only in the activation energy of exchange $E_{\text {ex }}$ for $\mathrm{Ge}$ and $\mathrm{Si}$ parameters.

It should be emphasized that in our study we concentrated on the qualitative scaling behavior and did not intend to reproduce quantitative features. Therefore, the parameters listed above cannot be regarded as a fit of the DDP model to the experimental data. Still, a considerable agreement with the experiment is achieved.

In Figs. 4 and 5 results of the KMC simulation of the DDP model are presented. Simulations were performed at $F=0.2 \mathrm{BL} / \mathrm{s}, T=320 \mathrm{~K}$ for Ge parameters, and $T=400 \mathrm{~K}$ for $\mathrm{Si}$ parameters. At these temperatures, the model yields the island density that differs by less than $10 \%$ for both materials. As in the experiment, to obtain similar densities of $\mathrm{Ge}$ and $\mathrm{Si}$ islands, a lower temperature in $\mathrm{Ge}$ growth than in $\mathrm{Si}$ growth must be used.

Figures 4(a) and 4(b) show the morphologies obtained from the simulations with the two sets of parameters. In agreement with experiment, a more regular pattern of islands is observed with Si parameters.

Figures 4(c) and 4(d) show the rescaled island size distribution $N_{s}$ obtained from simulations with Ge and Si parameters at different $\theta$. Scaling of $N_{s}$ with $\theta$, characterized by a decreasing scaling function for Ge parameters at $320 \mathrm{~K}$ and a normal peaked scaling function for Si parameters at $400 \mathrm{~K}$ is obtained in agreement with experiment. Simulations at complementary temperatures of $380 \mathrm{~K}$ for Ge and $340 \mathrm{~K}$ for Si reveal that a normal scaling behavior with a peaked size distribution of $\mathrm{Ge}$ islands is recovered with the increasing $T$. For Si parameters, no significant change of $N_{s}$ within the temperature interval comparable to the experimental one is observed.

Figures 4(e) and 4(f) show the rescaled nearest-neighbor separation distribution of islands $N(r)$ obtained from simulations with $\mathrm{Ge}$ and $\mathrm{Si}$ parameters. In agreement with the experiment, the rescaled $N(r)$ for Ge parameters is biased towards small island separations, while for Si parameters, the rescaled $N(s)$ is symmetric. This indicates nucleation of islands at smaller relative separations in the simulation with Ge parameters than with Si parameters. The appearance of the denuded zones has also been reproduced correctly in the
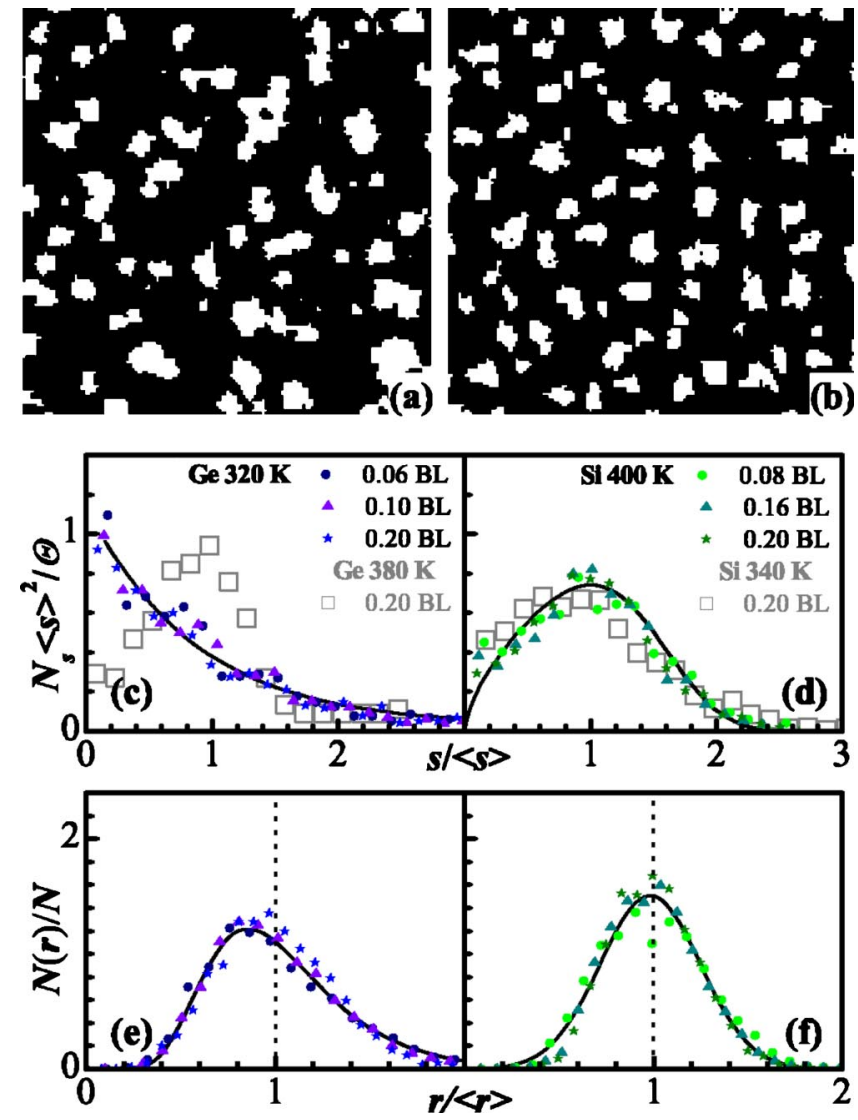

FIG. 4. (Color online) Morphology obtained from KMC simulations of the DDP model of SME. (a) Ge parameters, (b) Si parameters. $\theta=0.2 \mathrm{BL}, F=0.2 \mathrm{BL} / \mathrm{s} . T=320 \mathrm{~K}$ for $\mathrm{Ge}, T=400 \mathrm{~K}$ for $\mathrm{Si}$. Image width is 150 lattice units (of 512). (c), (d) Corresponding plots of the rescaled $N_{s}$ from simulation at various $\theta$ (closed symbols), simulation of $N_{s}$ for complementary temperatures (open symbols). (e), (f) Corresponding rescaled nearest neighbor separation distribution $N(r)$ of islands obtained from simulation for $\mathrm{Ge}$ and $\mathrm{Si}$ parameters. Lines represent guides to the eye (Ref. 42).

simulation (Fig. 5), showing no denuded zone for Ge parameters [Fig. 5(a)], and a pronounced denuded zone for Si parameters [Fig. 5(b)].

Finally, values of $\chi$ have been measured in the simulation for Ge parameters at $320 \mathrm{~K}$ and $\mathrm{Si}$ parameters at $340 \mathrm{~K} . \chi$
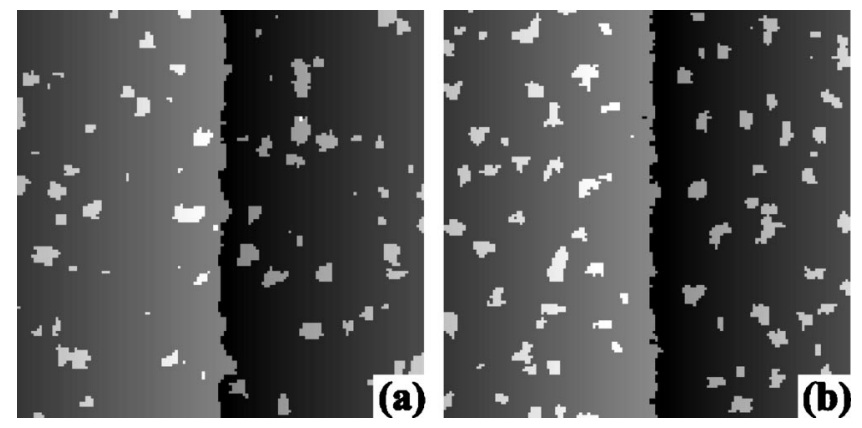

FIG. 5. Island population near a step edge as obtained from KMC simulations of the DDP model of SME. (a) Ge parameters, (b) Si parameters. $\theta=0.1 \mathrm{BL}, F=0.2 \mathrm{BL} / \mathrm{s} . T=320 \mathrm{~K}$ for $\mathrm{Ge}, T$ $=440 \mathrm{~K}$ for $\mathrm{Si}$. Image width is 150 lattice units (of 256). 
was determined from a dependence of $N$ on $F$ for 5 values of $F$ between $0.1 \mathrm{BL} / \mathrm{s}$ and $2 \mathrm{BL} / \mathrm{s}$. We obtain $\chi_{\mathrm{Ge}}^{320 \mathrm{~K}}$ $=0.41 \pm 0.02$ in good agreement with experiment. $\chi_{\mathrm{Si}}^{340 \mathrm{~K}}=0.28 \pm 0.02$ underestimates the related experimental value, however, is still near the lower limit predicted theoretically for $\chi$ in the standard growth model [Eq. (2)]. ${ }^{38}$

\section{DISCUSSION}

Results of our KMC simulations of SME allow to formulate new implications of the DDP model. Particularly, under certain conditions, the DDP model yields a nonstandard decreasing scaling function for the distribution $N_{s}$ of submonolayer island sizes. This nonstandard scaling behavior is temperature dependent: at higher temperatures, the island size distribution $N_{s}$ obeys the normal scaling with a peak at $s$ $=\langle s\rangle$. The appearance of the decaying scaling function in the DDP model is accompanied by the narrowing of denuded zones around step edges and islands.

In order to understand the origin of the nonstandard scaling behavior we recall that it was observed in our simulations using the $\mathrm{Ge}$ parameter set only, while with the $\mathrm{Si}$ parameters the standard peaked scaling function was reproduced at all considered temperatures. The main difference of the two sets of parameters is the value of the activation energy $E_{\text {ex }}$ needed for incorporation of the deposited atoms below the surfactant layer via the exchange process. In comparison with the barrier to diffusion of deposited atoms on top of the surfactant $E_{\text {ex }}(\mathrm{Ge})>E_{\mathrm{hop}}(\mathrm{Ge})$ for $\mathrm{Ge}$, while $E_{\text {ex }}(\mathrm{Si})=E_{\text {hop }}(\mathrm{Si})$ for $\mathrm{Si}$. This means that almost every hop of a $\mathrm{Si}$ atom on top of the surfactant is followed by an exchange/de-exchange event. Therefore, $\mathrm{Si}$ atoms reaching the step edge position will exchange and attach to the Si step edge with a high probability. On the contrary, Ge atoms on top of the surfactant make many hops before an exchange/ de-exchange event $(\approx 5000$ hops before an exchange event for single atoms and Ge parameters at $320 \mathrm{~K}$ ). This causes that $\mathrm{Ge}$ atoms reaching the step edge position can leave this position with much higher probability than to exchange and attach to the step edge. In other words, in the DDP model the step edges with the Ge parameter set are passivated much stronger than with the Si parameter set. Therefore, the nonstandard scaling phenomena in the DDP model can be regarded as a result of the strong passivation of step edges in the presence of surfactant.

The main consequence of the strong step edge passivation is the transition from growth limited by the surface diffusion to growth limited by the attachment kinetics. ${ }^{39}$ In the latter case the adatom distribution between step edges becomes uniform. ${ }^{39}$ Therefore the nucleation of new islands occurs with equal probability across the surface and no correlation in the island locations is observed. In this situation the nucleation kinetics resembles in many respects the nucleation ki- netics in the Kolmogorov-Avrami model, ${ }^{40,41}$ which, indeed, predicts a decreasing power-law scaling function for island sizes. ${ }^{27}$ The asymmetric shape of the nearest-neighbor separation distribution $N(r)$ as observed in our experiments for Ge islands was also proposed to be a signature of the growth mode with the uniform adatom density. ${ }^{27}$

Another signature of the growth mediated by the incorporation kinetics could be a flux dependence of the total island density with the exponent $\chi>1 .{ }^{13}$ However, both for $\mathrm{Si}$ and Ge our study revealed $\chi<0.5$, i.e, lower than the lower limit predicted for this case by the theory. ${ }^{13}$ This shows that the island size distribution is more sensitive to the actual regime of growth than the total island density.

As the deposition temperature increases the role of the exchange barrier vanishes and the kinetic regime of the island growth can change to the diffusion one. ${ }^{39}$ In accord, the standard peaked island size distribution recovers, as observed in our experiments and reproduced by KMC simulations. A similar temperature transition from a decreasing scaling function for $N_{s}$ to a standard one was predicted for epitaxy with desorption of atoms from the surface. ${ }^{15,16}$ However, the decreasing scaling function occurs at high temperatures in this case. This is caused by the fact that the desorption influences the adatom density that becomes constant at high temperatures. ${ }^{16}$

\section{CONCLUSIONS}

In this work, we present the first experimental study of submonolayer island size distributions in surfactant mediated epitaxy (SME). We find scaling of island sizes to a standard scaling function in Bi-mediated SME of $\mathrm{Si}$ on $\mathrm{Si}(111)$. On the contrary, distribution of island sizes in Bi-mediated SME of $\mathrm{Ge}$ on $\mathrm{Si}(111)$ scales to a nonstandard scaling function with a peak that is strongly shifted towards small island sizes. This nonstandard scaling in Ge SME is temperature dependent. For higher temperatures, the scaling function approaches the standard one.

Using kinetic Monte Carlo simulations, we demonstrate, that the observed scaling phenomena can be explained considering exchange and de-exchange processes of $\mathrm{Ge}$ and $\mathrm{Si}$ atoms with the surfactant layer and a passivation of step edges on the $\mathrm{Si}(111)$ surface due to the presence of surfactant. ${ }^{23,24}$ The different scaling phenomena in Bimediated SME of Ge and $\mathrm{Si}$ on $\mathrm{Si}(111)$ can be explained considering a stronger passivation of step edges for the Ge atoms on the Bi-terminated $\mathrm{Si}(111)$ surface than for $\mathrm{Si}$ atoms.

\section{ACKNOWLEDGMENTS}

We would like to thank Neelima Paul for fruitful discussions and Helmut Stollwerk and Peter Coenen for the technical assistance. The stay of S.F. and J.M. in Germany was supported by the Alexander von Humboldt Foundation. 
*Electronic address: b.voigtlaender@fz-juelich.de

${ }^{1}$ A. Pimpinelli and J. Villain, Physics of Crystal Growth (Cambridge University Press, Cambridge, 1998).

${ }^{2}$ M.C. Bartelt and J.W. Evans, Phys. Rev. B 46, 12675 (1992).

${ }^{3}$ C. Ratsch, A. Zangwill, P. Šmilauer, and D.D. Vvedensky, Phys. Rev. Lett. 72, 3194 (1994).

${ }^{4}$ C. Ratsch, P. Šmilauer, A. Zangwill, and D.D. Vvedensky, Surf. Sci. 329, L599 (1995).

${ }^{5}$ J. Amar and F. Family, Phys. Rev. Lett. 74, 2066 (1995).

${ }^{6}$ J.A. Stroscio and D.T. Pierce, Phys. Rev. B 49, R8522 (1994).

${ }^{7}$ H. Brune, G.S. Bales, J. Jacobsen, C. Boragno, and K. Kern, Phys. Rev. B 60, 5991 (1999).

${ }^{8}$ B. Müller, L. Nedelmann, B. Fischer, H. Brune, and K. Kern, Phys. Rev. B 54, 17858 (1996).

${ }^{9}$ D.D. Chambliss and K.E. Johnson, Phys. Rev. B 50, 5012 (1994).

${ }^{10}$ A. Zangwill and E. Kaxiras, Surf. Sci. 326, L483 (1995).

${ }^{11}$ B.A. Joyce, D.D. Vvedensky, A.R. Avery, J.G. Belk, H.T. Dobbs, and T.S. Jones, Appl. Surf. Sci. 98, 357 (1998).

${ }^{12}$ L. Andersohn, Th. Berke, U. Köhler, and B. Voigtländer, J. Vac. Sci. Technol. A 14, 312 (1996).

${ }^{13}$ D. Kandel, Phys. Rev. Lett. 78, 499 (1997).

${ }^{14}$ B. Voigtländer, M. Kästner, and P. Šmilauer, Phys. Rev. Lett. 81, 858 (1998).

${ }^{15}$ P. Jensen, H. Larralde, and A. Pimpinelli, Phys. Rev. B 55, 2556 (1997).

${ }^{16}$ P.A. Mulheran and D.A. Robbie, Philos. Mag. B 80, 1299 (2000).

${ }^{17}$ M. Copel, M.C. Reuter, E. Kaxiras, and R.M. Tromp, Phys. Rev. Lett. 63, 632 (1989).

${ }^{18}$ M. Horn-von Hoegen, F.K. LeGoues, M. Copel, M.C. Reuter, and R.M. Tromp, Phys. Rev. Lett. 67, 1130 (1991).

${ }^{19}$ G. Meyer, B. Voigtländer, and N.A. Amer, Surf. Sci. 274, L541 (1992).

${ }^{20}$ B. Voigtländer and A. Zinner, J. Vac. Sci. Technol. A 12, 1932 (1994).

${ }^{21}$ B. Voigtländer, A. Zinner, T. Weber, and H.P. Bonzel, Phys. Rev. B 51, 7583 (1995).

${ }^{22}$ I.-S. Hwang, T.-C. Chang, and T.T. Tsong, Phys. Rev. Lett. 80, 4229 (1998).

${ }^{23}$ D. Kandel and E. Kaxiras, Phys. Rev. Lett. 75, 2742 (1995).
${ }^{24}$ D. Kandel and E. Kaxiras, Solid State Phys. 54, 219 (2000).

${ }^{25}$ For the evaluation of $N_{s}$, areas on the surface not influenced by step edges, domain boundaries and $\mathrm{Si}$ islands created during $\mathrm{Bi}$ termination were selected.

${ }^{26}$ C. Ratsch, M.F. Gyure, S. Chen, M. Kang, and D.D. Vvedensky, Phys. Rev. B 61, R10598 (2000).

${ }^{27}$ P.A. Mulheran and D.A. Robbie, Philos. Mag. Lett. 78, 247 (1998).

${ }^{28}$ J.A. Venables, Surf. Sci. 299, 798 (1994).

${ }^{29}$ V. Bressler-Hill, S. Varma, A. Lorke, B.Z. Nosho, P.M. Petroff, and W.H. Weinberg, Phys. Rev. Lett. 74, 3209 (1995).

${ }^{30}$ B.Z. Nosho, V. Bressler-Hill, S. Varma, and W.H. Weinberg, Surf. Sci. 364, 164 (1996).

${ }^{31}$ C. Ratsch, A. Zangwill, and P. Šmilauer, Surf. Sci. 314, L937 (1994).

${ }^{32}$ S. Tan and P.-M. Lam, Phys. Rev. B 59, 5871 (1999).

${ }^{33}$ P. Šmilauer, M.R. Wilby, and D.D. Vvedensky, Phys. Rev. B 47, 4119 (1993).

${ }^{34}$ A.B. Bortz, M.H. Kalos, and J.L. Lebowitz, J. Comput. Phys. 17, 10 (1975).

${ }^{35}$ K. Schroeder, A. Antons, R. Berger, and S. Blügel, Phys. Rev. Lett. 88, 046101 (2002).

${ }^{36}$ K. Schroeder (private communication); K. Schroeder, A. Antons, R. Berger, and S. Blügel, Phase Transitions 75, 91 (2002).

${ }^{37} E_{n}^{\text {inc }}>E_{n}^{\text {top }}$ ensures that virtually all deposited atoms incorporate below surfactant during growth.

${ }^{38} \mathrm{An}$ increase of simulated $\chi_{\mathrm{Si}}^{340 \mathrm{~K}}$ can be obtained by lowering $E_{n}^{\mathrm{inc}}$ in Si parameters, however, on the costs of readjusting other $\mathrm{Si}$ parameters to obtain an agreement with other experimental data. The concern of this work is to keep $\mathrm{Si}$ and Ge parameter sets as similar as possible.

${ }^{39}$ I. Markov, Surf. Sci. 429, 102 (1999).

${ }^{40}$ A.N. Kolmogorov, Bull. Acad. Sci. USSR, Phys. Ser. (Engl. Transl.) 1, 335 (1937).

${ }^{41}$ M. Avrami, J. Chem. Phys. 7, 1103 (1939).

${ }^{42}$ The lines are (c) a double exponential, (d) an analytical expression for rescaled $N_{s}$ proposed in Ref. 5, (e) a log-normal distribution, and (f) a normal distribution. 